\title{
CHOLECYSTECTOMIES AT RIMS, ONGOLE- A RETROSPECTIVE ANALYSIS
}

\author{
Sistla V. P. L. Chandrakumar1, Tatikonda Suman²
}

${ }^{1}$ Associate Professor, Department of Surgery, RIMS, Ongole.

2Senior Resident, RIMS, Ongole.

ABSTRACT
BACKGROUND
Gallstones are one of the most common medical problems leading to surgical intervention throughout the world. At least $10 \%$ of
the adults have gallstones with a recent rise in the incidence due to westernisation of diet. It has been well demonstrated that the
presence of gallstones increases with age. During the reproductive age, the female-to-male ratio is about $4: 1$. Laparoscopic
cholecystectomy is one of the most common operation performed worldwide. In most cases, this operation is safe. But
complications do occur which range from simple wound infection to major biliary tract injuries adding significantly to morbidity
and mortality. This study has been carried out with an objective of retrospective analysis of cholecystectomies conducted at Rajiv
Gandhi Institute of Medical Sciences, Ongole, for a period of 1 year from July 2016 to July 2017 .
The aim of this study is to review the epidemiology with respect to the age and sex distribution of patients who underwent
cholecystectomies at RIMS, Ongole, during the period between July 2016 and June 2017 , to review the conversion rate from
laparoscopy to open surgery, to review the complications and the final histopathology report.

\section{MATERIALS AND METHODS}

All cholecystectomies performed in RIMS, Ongole, during the period from July 2016 and June 2017, are reviewed. A total of 56 cases were studied with respect to age, sex distribution, solitary vs multiple stone disease, conversion rate, operating time, complications and histopathology report. The indication/s for surgery itself is not part of the study, neither the preoperative symptoms nor the duration of symptoms.

\section{RESULTS}

From June 2016 to July 2017, a total of 56 cases of cholecystectomies were conducted in our hospital. Contrary to the popular belief in our series cholecystectomies were more common in males ( 32 males vs 24 females), the maximum cholecystectomies were performed for patients in their fifth decade even though no age is exempted from the disease process. Sonography was the investigation of choice in all cases with the most common finding being multiple gall stones, contracted gall bladder. Large solitary stone was relatively less frequent. The conversion from laparoscopy to open was 8\% (4 cases out of 50) and 6 cases were direct open cholecystectomies due to contraindications for laparoscopy and most suggestive histopathology was chronic cholecystitis. Clearly laparoscopic cholecystectomy was better than open procedure with respect to postoperative pain, faster return to activity and hospital stay.

\section{CONCLUSION}

There are several studies which suggest male dominant incidence of cholelithiasis, of which ours is one. Because the number of cases is relatively small, a much large series with randomisation is necessary to prove/ disprove other results of our study.

\section{KEYWORDS}

Gallbladder, Cholelithiasis, Cholecystitis, Cholecystectomy, Laparoscopy, Bile Duct Injury.

HOW TO CITE THIS ARTICLE: Chandrakumar SVPL, Suman T. Cholecystectomies at RIMS, Ongole- a retrospective analysis. J. Evolution Med. Dent. Sci. 2017;6(68):4890-4893, DOI: 10.14260/Jemds/2017/1060

\section{BACKGROUND}

Cholelithiasis is a relatively common clinical condition, which over time has become the bread and butter for the laparoscopic surgeon. Laparoscopic cholecystectomy is one of the most common operation performed worldwide. In our hospital, we performed about 56 cases of laparoscopic cholecystectomies during the past one year. We made an attempt to review all those cases so that we have an idea about the epidemiology of the disease itself, our performance

Financial or Other, Competing Interest: None.

Submission 20-07-2017, Peer Review 12-08-2017,

Acceptance 19-08-2017, Published 24-08-2017.

Corresponding Author:

Dr. Sistla V. P. L. Chandrakumar,

Department of General Surgery,

RIMS, Ongole-523001, Andhra Pradesh.

E-mail: chandrakumarsistla@gmail.com

DOI: $10.14260 /$ jemds/2017/1060 indices like operating time on average, conversion rate and complication rate. This paper is the result of this study and provides data to improve the performance of our institute.

\section{MATERIALS AND METHODS}

The study constitutes a retrospective analysis of cholecystectomies conducted at Rajiv Gandhi Institute of Medical Sciences, Ongole, for a period of 1 year from July 2016 to July 2017. All patients were investigated for routine blood investigations. USG of abdomen was the baseline investigation for confirmation of diagnosis. All patients posted for laparoscopic surgery were explained in detail about the procedure and about possible conversion to open cholecystectomy when needed. A well-informed, explained and written consent was taken of all patients who were posted for laparoscopic cholecystectomy. All patients were operated under general anaesthesia. Detailed history of all the 56 cases were taken according to the proforma. 
Information regarding the age, religion, socioeconomic status, nature of the symptoms, duration of the symptoms, past history of similar complaints, diet history, history of OC pills, alcohol ingestion and diabetes was obtained. All patients had undergone detailed examination. All patients had haemogram, ECG, LFT, blood sugar, blood urea, serum creatinine, urine analysis, blood group and chest x-ray, ultrasound scan of the abdomen. Relevant investigations and speciality consultations were taken for patients with associated medical illness and their control was achieved. Risk and complications of the condition as well as surgery was explained to the patient, consent was taken. Patient was properly examined in the postoperative period to note the development of any complication. Suitable treatment was given according to the need. Patients were advised regarding diet, rest and to visit the surgical OPD for regular followup. In the followup period, attention were given to subject for improvement of the patients with regard to symptoms as well as examination of the operative scar.

\section{RESULTS}

A total of 56 cases that were studied retrospectively over a period of 1 year that were treated as inpatients basis at Rajiv Gandhi Institute of Medical Sciences, Ongole, from July 2016 to July 2017. In my study, the youngest patient was 12 years old and the oldest patient is 71 years old. There is an increased incidence in the $5^{\text {th }}$ and $6^{\text {th }}$ decade with the maximum incidence in the $5^{\text {th }}$ decade (35\% of patients) and least incidence in $2^{\text {nd }}$ decade (1.7\% of patients). Whereas $3^{\text {rd }}$ decade shows $7.14 \%$ of patients, $4^{\text {th }}$ decade shows $21.4 \%$ of patients. In the present study 32 out of 56 cases were male, while the rest 24 were female. Female-to-male ratio is $3: 4$. Even though the literature suggests a female dominance in the incidence of cholelithiasis, in our study the number of males undergoing cholecystectomy for gall stones pared the female incidence. The 46 patients were moderately built and nourished, 10 patients were obese. These observations are against the time-honoured aphorism that fat people are more prone for this disease. Majority of our patients were nondiabetic. The indication for cholecystectomy was symptomatic gall stones rather than an acute attack. Ultrasound scanning was done in all patients. All the cases revealed stone in the gallbladder. $28.5 \%$ (16 patients) had solitary stones in gall bladder, $82 \%$ (40 patients) had multiple stones in gallbladder. Contracted gall bladder was present in $85.7 \%$ (48 patients) of the cases. In the present study 50 patients underwent lap cholecystectomy $(89.2 \%$ of patients). The conversion rate from lap to open cholecystectomy was $8 \%$ (4 out of 50 cases). Conversion from lap to open cholecystectomy is because of bleeding from cystic artery in one case, frozen Calot's in one case and bile duct injury in two cases. Open cholecystectomy was done in 6 cases, as they were unfit for laparoscopic cholecystectomy because of COPD. In all open cholecystectomy cases, duct first method was done except in one case where fundus first method was done. The reason for fundus first method was dense adhesions, difficulty in identifying Calot's triangle. The duct first method was the method of choice. The operative room time for lap cholecystectomy was ranged from 88 mins. to 126 mins. with approximate average time being 103 mins. In the present study wound infection was the most common complication, which was seen in $5.3 \%$ of cases $(2$ cases of open cholecystectomy and 1 case of lap cholecystectomy). Haemorrhage in $5.3 \%$ of cases (3 cases of lap cholecystectomy), 2 patients had bile leakage through the drain tube in lap cholecystectomy group, the patient was managed conservatively and the patient improved. In this case, drain was removed on the $7^{\text {th }}$ day. Prolonged ileus was seen in one case. Two cases in lap cholecystectomy and one case in open cholecystectomy had bile duct injury in my study. Bile duct injury is due to difficulty in dissecting the Calot's triangle. In the present study, 50 patients were reported as having chronic cholecystitis (89.2\% of patients) and 6 patients were reported as having acute cholecystitis (10.7\% of patients). One patient had gangrenous gall bladder. There was no problem in the followup period in any patient. There is no history of recurrence, new or persistent of symptoms or biliary stricture of biliary fistula formation. There is no mortality in our series. Because most of the surgeons in our hospital are well trained, the conversion rate has been low.

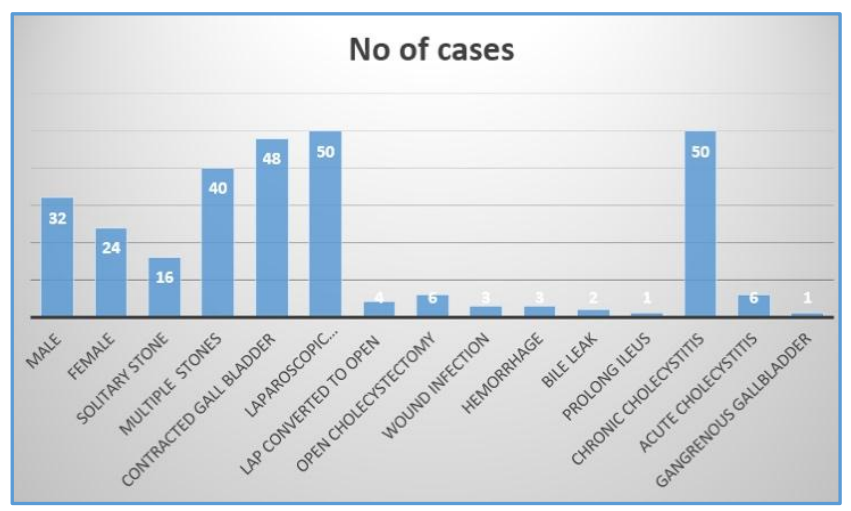

\section{DISCUSSION}

Most of the progress in the diagnosis and treatment of biliary tract disease has been made in the last century, but gall stones and their sequel which cause most of the maladies date back to 1085 - $945 \mathrm{BC}$ having been discovered in the mummy of Priestess of Amen. The first systematic data about the disease was published as "De Medical Historic Mirabilis" by Marcellus Donatus in 1596. Zambeccari in 1636 performed cholecystectomy in a dog. Herlin (1767 AD) a Frenchman performed surgery on experimental animals. He removed the gallbladder of a cat, which recovered well after the operation. The first cholecystectomy in man is credited to John Strong Bobbs on June 15, 1867. The first report of SILS cholecystectomy came in 1997 in a letter to the editor in the British Journal of Surgery by Navarra. In 1999, Piskun performed a SILS cholecystectomy by placing two $5-\mathrm{mm}$ trocars through a common umbilical incision and using transabdominal sutures to manipulate the gallbladder. The fascial bridge between the two trocars was then joined and the specimen extracted through this single umbilical incision. In early March 2007, the NOTES Research Group in Rio de Janeiro, Brazil, led by Dr. Ricardo Zorron, performed the first series of transvaginal NOTES cholecystectomy in four patients based on previous experimental studies. On June 25, 2007, Swanstrom and colleagues reported the first human transgastric cholecystectomy. Cadiere and colleagues reported the first successful clinical implementation of telerobotics in 1998 when they accomplished a laparoscopic 
cholecystectomy using a prototype of the Da Vinci robotic surgical system. Cholelithiasis is a common surgical problem, which makes cholecystectomy one of the most frequently performed surgical procedures. Its prevalence varies widely around the world. In India, it is around $4 \%$. An epidemiological study restricted to rail road workers showed that north Indians have 7 times higher occurrence of gall stone as compared with south Indians. There is a significantly increased risk of cardiovascular disease among patients with gallstone disease. ${ }^{1}$ Gallstone disease is associated with increased mortality in the United States. ${ }^{2}$ Genetic and functional identification of the likely causative variant for cholesterol gallstone disease is at the ABCG5/8 lithogenic locus. ${ }^{3}$ The prevalence of gallbladder stones is higher among patients with chronic kidney disease in Taiwan. ${ }^{4}$ Susruta described jaundice called pittaash mari janya meaning a jaundice caused by stone in bile. ${ }^{5}$ Cholecystectomy is the preferred treatment for symptomatic gallstones. However, approximately half the observation group did not require surgery or suffered complications indicating that it may be a valid alternative to surgery. ${ }^{6}$ Gallstone disease is strongly associated with CAD diagnosed by coronary angiography. ${ }^{7}$ In the United States, the autopsy series have shown gall stones in at least $20 \%$ of women and $8 \%$ of men over the age of 40 years. Changing incidence in India is mainly attributed to westernisation and availability of investigation that is ultrasound to urban as well as rural area and also because of increased affordability due to change in the socio-economic structure and the cost of investigations. Because of increased incidence of gall stones and its variable presentations in India as well as in the west, there is a great need for a study which can provide the information regarding the prevalence of the disease, management, outcomes of the cholelithiasis. Currently, one of the contraindication for laparoscopic cholecystectomy is precluding safe portal dissection. ${ }^{8}$ Anatomical variations of the hepatic and cystic arteries are seen in approximately 50\% of individuals. 9,10 Elevated body mass index as a causal risk factor for symptomatic gallstone disease, a Mendelian randomisation study. ${ }^{11}$ Gallbladder sludge is the precursor, mucoprotein is a major component of gall stone. Extreme bilirubin levels is a causal risk factor for symptomatic gallstone disease. Gallstones seem to increase the risk of both intra- and extra-hepatic cholangiocarcinoma. However, this risk seems to decline to the level of the background population with time after cholecystectomy. The introduction of laparoscopic cholecystectomy has emerged as the gold standard for the treatment of patients with symptomatic gall stones. It is being performed as a day care surgery, thus saving on human and hospital working hours. The advantage of laparoscopic cholecystectomy is greater, especially in developing countries where demands for hospital bed are great and early return to work a financial necessity. Laparoscopic cholecystectomy is a newly developed technique that avoids the traditional upper abdominal incision, diminished postoperative pain, more rapid recovery with full return to activity and superior cosmetic results. Patel and Veverka 2002 showed that laparoscopic cholecystectomy can be performed safely in pregnant patient in the first two trimesters; while open cholecystectomy should be considered the procedure of choice in patient with severe cholecystitis, empyema of gall bladder, acute cholangitis, gall bladder perforation, cholecystenteric fistula or a suspected gall bladder neoplasm. It has been observed that intraoperative findings such as adhesions in Calot's triangle, adhesions between gall bladder and duodenum, relation of gall bladder with liver i.e. due to repeated inflammation plane between gall bladder and liver is obliterated and developmental anomalies which are easily missed on ultrasonography have a great influence on the outcome of the surgery in terms of increased operative time and hence risk of anaesthesia and $\mathrm{CO} 2$ toxicity. The only disadvantage of lap cholecystectomy over open procedure is the duration of operating time which is significantly longer, especially when the Calot is frozen or the patient is obese and surgeon works with regular instruments. Complications associated with laparoscopic cholecystectomy as observed are haemorrhage, bile duct injury, bile leak, ruptured gall bladder and spilled gall stones during laparoscopic cholecystectomy, acute postoperative pancreatitis (due to migration of stone), wound infection, incisional hernia, pneumoperitoneum related complications like $\mathrm{CO} 2$ embolism, vasovagal reflex, cardiac arrhythmias, hypercarbic acidosis, trocar related abdominal wall bleeding, haematoma, visceral injury, vascular injury, etc. are also known. With the advent of laparoscopy, the rate of serious bile duct injuries after cholecystectomy had a discreet increase of up to $0.8 \%$, whilst the one related to open route remained between 0.2 0.3 percent. The prognosis is directly related to the patient's underlying conditions as well as the time elapsed between the lesion and its identification and treatment, which is basically the reconstruction of the biliary path. The Roux-en$\mathrm{Y}$ hepaticojejunostomy is considered to be the treatment of choice today. Currently, one of the contraindication for laparoscopic cholecystectomy is precluding safe portal dissection. In the United States alone, the diagnosis and treatment of gallstone disease accounts for more than $\$ 5$ billion in direct costs including about half a million cholecystectomies. There is an increasing prevalence with age after the age of 60 about $10 \%$ to $15 \%$ of men and $20 \%$ to $40 \%$ of women have gallstones. Gallbladder atony consequent upon an autonomic neuropathy may favour gall stones. Diabetes patients are prone to complications. Symptomatic gallstone forms in approximately $45 \%$ of patients who are maintained on long-term TPN. Patients with ileal dysfunction which is more saturated with cholesterol and patients with jejunoileal operation are associated with increased risk.

\section{Pathogenesis of Gall Stones}

Gall stones are composed mainly of cholesterol, bilirubin and calcium. Pure cholesterol crystals are quite soft. Noncholesterol stones are black or brown pigment stones. Primary brown pigment stones are found in decreased biliary secretory IgA. Cholesterol is virtually insoluble in aqueous solution, but in bile it is made soluble by association with bile salts and phospholipids in the form of mixed micelles and vesicles. The molar proportions of cholesterol, phospholipids and bile acids in bile are often represented on triangular coordinates. Gallbladder sludge is the precursor, mucoprotein 
is a major component. Extreme bilirubin levels are as a causal risk factor for symptomatic gallstone disease.

\section{CLINICAL MANIFESTATIONS}

\section{Presentation}

Two thirds of gall stones are asymptomatic. Stones may cause acute or chronic cholecystitis, biliary colic, pancreatitis or obstructive jaundice. The patient might experience episodes of acute abdominal pain called biliary colic. Dropped gallstones may rarely become symptomatic causing recurrent abscesses. Diagnosis is challenging due to unusual clinical presentations, myriad locations and radiologically occult calculi. Out of sight, but kept in mind complications and imitations of dropped gall stones.

\section{Obstructive Jaundice}

This occurs when a stone migrates from the gall bladder into the common bile duct or less commonly when fibrosis and impaction of a large stone in Hartmann's pouch compresses the common hepatic duct (Mirizzi's syndrome).

\section{Physical Signs}

Positive Murphy's sign where in sitting position during deep inspiration, while palpating in right hypochondrium, patient winces with pain at the summit of the inspiration. Same sign elicited in lying down position is called as Moynihan's sign. Hyperesthesia between the $9^{\text {th }}$ and $11^{\text {th }}$ ribs posterior on the right side is present in acute cholecystitis called as Boas' sign. Complete or partial obstruction of the common bile duct manifests as jaundice.

\section{CONCLUSION}

The statistical issues in our study is on par with standard studies except with some disparity with respect to sex distribution. However, being a small observational study, it needs much larger randomised study for confirmation.

\section{REFERENCES}

[1] Olaiya MT, Chiou HY, Jeng JS, et al. Significantly increased risk of cardiovascular disease among patients with gallstone disease: a population-based cohort study. PLoS ONE 2013;8(10):e76448.
[2] Ruhl CE, Everhart JE. Gallstone disease is associated with increased mortality in the United States. Gastroenterology 2011;140(2):508-16.

[3] Von Kampen O, Buch S, Nothnagel M, et al. Genetic and functional identification of the likely causative variant for cholesterol gallstone disease at the ABCG5/8 lithogenic locus. Hepatolog 2013;57(6):2407-17.

[4] Lai SW, Liao KF, Lai HC, et al. The prevalence of gallbladder stones is higher among patients with chronic kidney disease in Taiwan. Medicine (Baltimore) 2009;88(1):46-51.

[5] Park K. Man and medicine towards health for all. 17th edn. Park's text book of preventive and social medicine. Banarasi das Pub 2002:1-10.

[6] Brazzelli M, Cruickshank M, Kilonzo M, et al. Systematic review of the clinical and cost effectiveness of cholecystectomy versus observation/conservative management for uncomplicated symptomatic gallstones or cholecystitis. Surg Endosc 2015;29(3):637-47.

[7] Jiang ZY, Sheng X, Xu CY, et al. Gallbladder gallstone disease is associated with newly diagnosed coronary artery atherosclerotic disease: a cross-sectional study. PLOS ONE 2013;8(9):e75400.

[8] Courtney M, Townsend, Beauchamp RD, et al. Biliary tract. $19^{\text {th }}$ edn. Sabiston text book of surgery. Elsevier Publication 2012:P 1491.

[9] Izbéki F, Rosztóczy AI, Yobuta JS, et al. Increased prevalence of gallstone disease and impaired gallbladder motility in patients with barrett's esophagus. Dig Dis Sci 2008;53(8):2268-75.

[10] Pierre-Alainclavien, Baillie J. Disease of the gallbladder and bile ducts: diagnosis and treatment. The new England journal of Medicine 2002;346:1593-4.

[11] Stender S, Nordestgaard BG, Tybjaerg-Hansen A. Elevated body mass index as a causal risk factor for symptomatic gallstone disease: a mendelian randomization study. Hepatology 2013;58(6): 2133-41. 